\title{
A chaotic time series prediction algorithm using asymmetric functions
}

\author{
Bu Yun, Kang Wan Xin, Chen Yong Qiang
}

School of Electrical and Information Engineering, Xihua University, Cheng Du, 610039

\begin{abstract}
To connect prediction functions with physical phenomena, we designed two shape-variable functions to improve the adaptability of a prediction algorithm for asymmetric distributed chaotic signals. An adjustable parameter controls the shape of the presented functions. In simulation, we proved that if the chaotic series are asymmetric, the best performance appears when the shape of the basis function is similar with the shape of density functions.
\end{abstract}

Keywords: Chaotic signal, adaptive prediction, probability density function, shape-variable function

\section{Introduction}

As a fundamental aspect of chaos theory, predicting the future trajectory is a challenging and an attractive task with widely applications, such as stock market returns and exchange rate ${ }^{[1]} . \mathrm{Up}$ to now, many prediction algorithms were presented and provided availability ${ }^{[2-5]}$. These reported methods were developed basing on different theories, including neural networks, fuzzy functions, Volterra series approaches and so on. To obtain an optimum prediction expression automatically, genetic algorithm based approaches were presented ${ }^{[6]}$, which can compensate for the incomplete acquisition or empirical bias of designers.

In principle, limitless functions can be used to construct a prediction algorithm.
However, their performances will be difference because of the different used functions and the complexity of chaotic systems. Then in reported papers, most endeavors were made to search novel basis functions to improve the performance of an algorithm. A preferred prediction model not only should trace the future trajectory accurately, but also have a direct correspondence with physical phenomena. But up to now, few approaches can satisfy both the requirements. In our previous works ${ }^{[7-8]}$, some attempt was made to connect the prediction model with statistic characters of a chaotic system. But these functions are so general that they can't show individuality of different systems. Moreover, this method does not supply a straightforward way to ascertain which function is suitable in advance. So we presented an improvement approach using functions that show similar shapes with the distribution function of a chaotic system to include both statistic information and the individuality.

\section{Shape-Variable Functions}

Chaotic systems show different density functions, but generally symmetric functions are popular to construct a prediction algorithm. To improve the adaptability of an algorithm, some shape-variable functions should be introduced into a prediction method. Then we designed two different shape variable functions, even function based and odd function based shape variable functions. 
The even function based variable geometric double sigmoid function is

$$
y(x)=0.5\left\{\tanh \left[\left(x-C_{1}\right) \times W_{1}\right]-\tanh \left[\left(x-C_{2}\right) \times W_{2}\right]\right\}
$$

where four parameters $\mathrm{C}_{1}, \mathrm{~W}_{1}, \mathrm{C}_{2}$ and $\mathrm{W}_{2}$ are positive, adjustable and they determine the wave of the function.

Usually, preferred input signals are within the range of -1 to 1 , thus we assigned $\mathrm{C}_{1}=\mathrm{C}_{2}=0.5$, and a variable shape parameter $\mathrm{W}$ :

$$
\begin{aligned}
& W_{1}=2-W \\
& W_{2}=2+W
\end{aligned}
$$

By changing the value of $\mathrm{W}$, the function curve will show corresponding variation, as shown in Fig. 1.

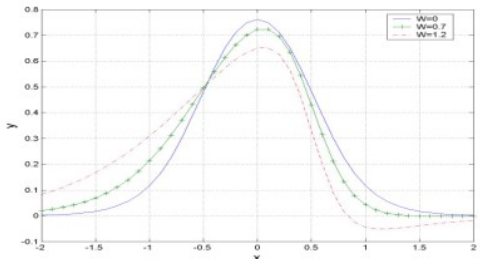

Fig. 1 Plots of the Eq. 1 for different values of W, $\mathrm{W}=0, \mathrm{~W}=0.7, \mathrm{~W}=1.2$.

We assigned three values for $\mathrm{W}$, $\mathrm{W}=0, \mathrm{~W}=0.7$ and $\mathrm{W}=1.2$. Figure 1 shows that when $\mathrm{W}=0$ (solid line), the function is symmetric and even. When $\mathrm{W}>0$, the curve is skewed toward positive direction, vice versa. Moreover, the larger the $\mathrm{W}$ is, the more asymmetry the curve is. Another corresponding odd function based shape-adaptive expression is defined as:

$$
y(x)=x\left\{\exp \left[-\left(x+C_{1}\right)^{2} \times W_{1}\right]+\exp \left[-\left(x-C_{2}\right)^{2} \times W_{2}\right]\right\}
$$

where $\mathrm{C}_{1}=\mathrm{C}_{2}=0.5, \mathrm{~W}_{1}=4+\mathrm{W}, \mathrm{W}_{2}=4-\mathrm{W}$.

The same as Eq. 1, Eq. 3 can show various shapes by changing the value of the parameter $W$. In the case of $W=0$, the function is odd symmetric. When W becomes larger than zero, the odd symmetric state is broken and the plot slants to the right. Fig. 2 outlines three different curves with $\mathrm{W}=0, \mathrm{~W}=0.7$ and $\mathrm{W}=1.2$, which indicate that the extent of asymmetry and the direction of skew are controlled by W.

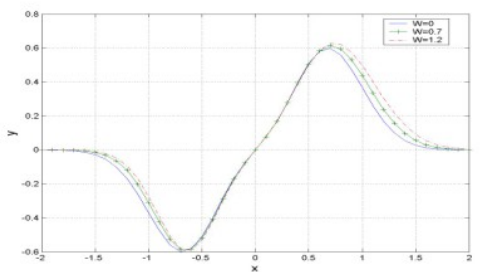

Fig. 2 Plots of the Eq. 4 for $\mathrm{W}=0 . \mathrm{W}=0.7, \mathrm{~W}=1.2$

\section{Adaptive Prediction Algorithms}

To reflect asymmetric distribution of some chaotic signals, we established adaptive prediction algorithms by introducing the aforementioned two shape variable functions as basis functions:

$$
y(n+1)=\sum_{i=1}^{N} \alpha_{i} f_{i}(W, \bar{X}(n))
$$

where $\alpha_{i}$ is coefficients, $f_{i}(\cdot)$ is a basis function with two parameters, W(n) and $\bar{X}(n)$. W dominates the shape of $f_{i}(\cdot)$, and $\bar{X}(n)$ is the input reconstructed chaotic signal vector with $d$ embedded dimension,

$\bar{X}(n)=[x(n), x(n-\tau), \cdots, x(n-(d-1) \tau)]$.

The two under test chaotic maps are discrete and asymmetric systems, coupled Logistic system and Henon map. Coupled Logistic system is:

$$
\begin{aligned}
& x(n+1)=4 A x(n)(1-x(n))+R x(n) y(n) \\
& y(n+1)=4 A y(n)(1-y(n))+R x(n) y(n)
\end{aligned}
$$

where $\mathrm{A}=0.7, \mathrm{R}=0.64$.

Henon map is simple and defined as:

$$
\begin{aligned}
& x(n+1)=1+y(n)-1.4 x^{2}(n) \\
& y(n+1)=0.3 x(n)
\end{aligned}
$$


Their Histograms are shown as Figure 3 (a) and (b). From the figures, we found they are asymmetric distributed and skew to right.

Coupled Logistic system is a complex hyperchaotic system with two positive maximum Lypunov indexes, and most prediction methods can't give a well performance. To enhance the predicta-bility of an algorithm, we designed a prediction method based on the presented algorithms [7-8], where the formula (1) is introduced as a basis function component:

$$
\begin{aligned}
& F(x)=\left\{f_{1}(x), f_{2}(x), f_{3}(x), f_{4}(x)\right\} \\
& f_{1}(x)=x \\
& f_{2}(x)=(1-\exp (-5 x)) /(1+\exp (-5 x)) \\
& f_{3}(x)=-\log (\cosh (x)) \\
& f_{4}(x)=0.5 \times\{\tanh [W \cdot(x+0.5)]-\tanh [W \cdot(x-0.5)]\}
\end{aligned}
$$

where $f_{4}(x)$ is the equation (1), and the other functions are symmetric.

The benchmark time series contain 5500 data produced by the equation (5), where the former 5000 data were used to achieve the coefficients, and the residuals were test data. All the data were normalized in advance:

$$
\begin{aligned}
& A=(\mathrm{maxx}(+) \quad \mathrm{m} \dot{x} \mathrm{n}( \\
& B=(\mathrm{maxx}(-) \quad \mathrm{m} \dot{x} \mathrm{n}( \\
& y=(x-A) / B
\end{aligned}
$$

In the training procedure, the standard recursive least squares (RLS) algorithm was used in both approaches, (8) and (9). Mean square error (MSE) was applied to quantify the algorithm performance:

$$
M S E=10 \log _{10}\left[\frac{\sum_{i=1}^{K} e^{2}(i)}{\sum_{i=1}^{K} x^{2}(i)}\right]
$$

where $e(i)$ is the prediction error.

The shape parameter $\mathrm{W}$ was assigned different values in advance, from -0.7 to
1.7 , to show its influence to the prediction performance in figure 4 .

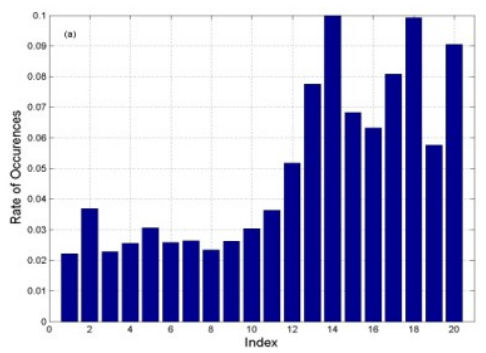

Fig. 3(a) Histograms of Coupled Logistic system

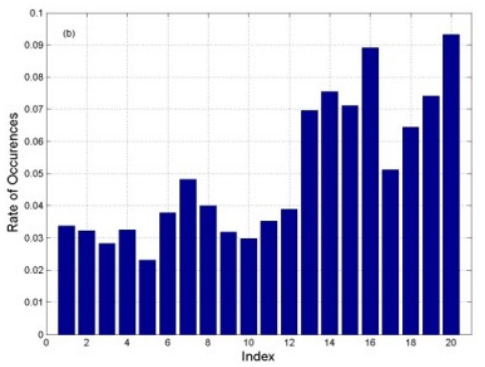

Fig. 3(b) Histograms of Henon map

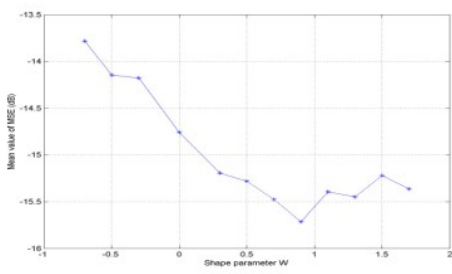

Fig. 4 Prediction performance versus shape parameter W for Coupled Logistic system.

Figure 4 describes the changes of mean MSE along the shape parameter W. It is clearly that the mean MSE curve shows a downward trend when the shape parameter $\mathrm{W}$ changes from -0.7 to 0.9 , and reached the bottom at $\mathrm{W}=0.9$, which indicates the best performance of the algorithm. When $\mathrm{W}$ increased to larger than 0.9, the curve shows a fluctuation, but the trend is upward. From the figure, we know the prediction performance is correlative with the shape of basis functions, namely the phenomena tallies 
with the right skew shape of the distribute function of Coupled Logistic system.

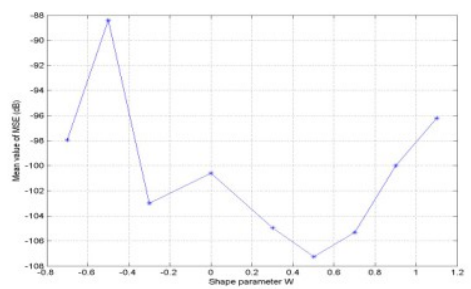

Fig. 5 Prediction performance versus shape parameter $\mathrm{W}$ for Henon map.

To prove the effect of the shape of a basis function to prediction accuracy, we applied another algorithm to Henon map and tested its performance. This algorithm has the basis function set $\left\{x, x^{2}, f(x)\right\}$, where $f(x)$ is defined by equation (2). Because Henon map is very simple, the length of training and testing data are 1000 and 500, respectively. Figure 5 is the simulation results, and it shows the mean MSE curve has a similar trend with the figure 4 . The best performance appeared when $\mathrm{W}=0.5$.

Both figure 4 and 5 illumine if the basis functions have the similar shape with the density function of under tested chaotic signals, the prediction performances would be enhanced. Obviously, in this case, the basis functions can supply more accuracy statistic information, which is corresponddence with our point of view in [7].

\section{Conclusion}

In this letter, we attempt to improve a prediction algorithm performance by using asymmetric basis functions that show similar shape with chaotic signals' density function. Two different shape-variable functions were designed to replace general odd and even functions respectively. In simulation, the superiority of this method over symmetric function based method was proved, and the optimum shapes were also funded.

\section{Acknowledgments}

This work is supported through Grants No. 12ZB132 and No. Z1120944, by the research foundations of the education bureau of Si Chuan Province and XiHua University.

\section{References}

[1]Shian-Chang Huang, Pei-Ju Chuang, Cheng-Feng $\mathrm{Wu}$ and Hiuen-Jiun Lai, Chaos-based support vector regress-ions for exchange rate forecasting, Expert Systems with Applications, 37, pp. 8590-8598, 2010

[2]Gong Xiaofeng, and C. H. Lai, Phys. Rev. E, Improvement of the local prediction of chaotic time series, Vol. 60, No. 5, pp. 5463-5468, Nov. 1999

[3]Min Han, Jianhui Xi, Shiguo Xu, and Fu-Liang Yin, Prediction of chaotic time series based on the recurrent predictor neural network, IEEE Trans. on Sig. Proc. Vol. 52, No. 2, pp. 3409-3416, Dec. 2004

[4]Zhang Jia-Shu, and Xiao Xian-Ci, Predicting chaotic time series using recurrent neural network, Chin. Phys. Lett. Vol. 17, No. 2, pp. 88-90, 2000

[5]D. Kim. Forecasting time series with genetic fuzzy predictor ensemble [J], IEEE Trans. on Fuzzy System, 1997, 5(4), 523-535.

[6]Vamsi K.Yadavalli, Rahul K.Dholes, Sanjeev S. Tambe, and B. D. Kulkarni, Obtaining functional form for chaotic time series evolution using genetic algorithm, Chaos, Vol. 9, No. 3, pp. 789-794, Sept. 1999

[7]BU Yun, WEN Guang-Jun, ZHOU Xiao-Jia, ZHANG Qiang. A novel adaptive predictor for chaotic time series. Chin. Phys. Lett. 2009, vol.26, No.10: 100502

[8]Yun BU, Guang-jun WEN, Hai-Yan JIN, Qiang ZHANG. Predictability of Iteration Method for Chaotic Time Series. IEICE Trans. on Fundamentals of Electronics, Communications and Computer Sciences, 2010, vol.E93-A, No.4: 840-842 